\title{
Power saving technique based on traffic channels reallocation in GSM mobile network
}

\author{
Vladimir Kosjer ${ }^{1}$, Dragan Mitić ${ }^{2}$, Aleksandar Lebl ${ }^{1}$, \\ Vladimir Matići ${ }^{1}$ Žarko Markov ${ }^{2}$
}

\begin{abstract}
A mobile users' network with power control on traffic channels, as it is GSM, is considered in this paper. It is analyzed the influence of busy channels reallocation on the necessary emission power. The main principle in reallocation is that users who need greatest power for their connections take traffic channels of the first carrier where power is always maximum. It is assessed that reallocation process is not more complicate than the simplest handover processes. It is proved that this method may be used to decrease emission power of carriers after the second one. Power saving depends on number of carriers, environmental propagation coefficient and users' density distribution in the cell. The results are verified by originally developed simulation program.

K e y w or ds: connection reallocation, power saving, frequency carriers, simulation program
\end{abstract}

\section{Introduction}

Energy saving in telecommunications is very important in order to find always new procedures to decrease necessary energy for one connection. The mobile users' network is the right example. The adaptive determination of necessary and sufficient power for the mobile users connection is called power control and it provides important results in energy saving. The base station (BTS) power limits at different transmission techniques implementation are presented in [1]. Base station power depends also on surface user density distribution in the network [2], on environmental attenuation coefficient [3], on the intra-cell connection part [4] as also on the user mobility [5].

The connection reallocation has been already used in classical switching systems [6] and the fundamental principles of these old networks are today implemented for fast re-routing (reallocation) in modern software-definednetworks in order to achieve load balancing and network resiliency in the case of some network element failure [7]. In networks with implemented VAMOS technology reallocation increases traffic capacity [8]. The reallocation is implemented in the case of systems with large number of micro-cells overlapped by one macro-cell [9]. The important role of reallocation between different layers of mobile telephony network in the increasing of traffic possibilities was noticed very early [10]. reallocation is also possible between base stations in the same hierarchical level [11] for the users situated in the overlapping area of two adjacent cells. Besides control of traffic overload, reallocation in this situation contributes to the avoidance of cell radius shrinking due to the increased interference in the third generation CDMA systems. But, the role of connections reallocation in decreasing base station power consumption is not analyzed in the available literature according to our knowledge. Contribution [12] considers power saving at mobile users as at BTS, but here power saving is achieved by rearranging transmitted packets structure, ie packet length. The cost for this method of power saving implementation is increased packet transmission delay and packet loss, thus decreasing connection quality. In our original paper it is investigated how BTS power may be saved by mobile users' connections reallocation.

We present main elements important for the model development. After allocation of channels in GSM mobile systems, which is the base for power saving algorithm implementation, is described, the power saving is realized by connections reallocation between different frequency carriers, according to below presented example. Ideal and real case of power saving are explained, and illustrated by an example. The three main factors (number of carriers, the value of environmental propagation coefficient and users density distribution in the cell), which have influence on the power saving rate, are investigated. Simulation programs related to the new technique are originally developed based on programs introduced in [2].

\section{Model, assumptions and designations}

Let us consider circular GSM cell, whose radius is $R$. There are $M$ users in the cell and $n$ carrier frequencies. The first frequency carrier always has maximum power. The total number of traffic channels $(\mathrm{TCH})$ is $N$. The BTS emission power for one connection is determined in accordance to the user's needs, ie it is proportional to the

\footnotetext{
${ }^{1}$ Department of Radio Communications Engineering, Institute IRITEL a.d., Belgrade 11080, Serbia, vladimir.kosjer@iritel.com, lebl@iritel.com, vmatic@iritel.com, ${ }^{2}$ Department of Optical Communications, Institute IRITEL a.d., Belgrade 11080, Serbia mita@iritel.com, zmarkov@iritel.com
} 


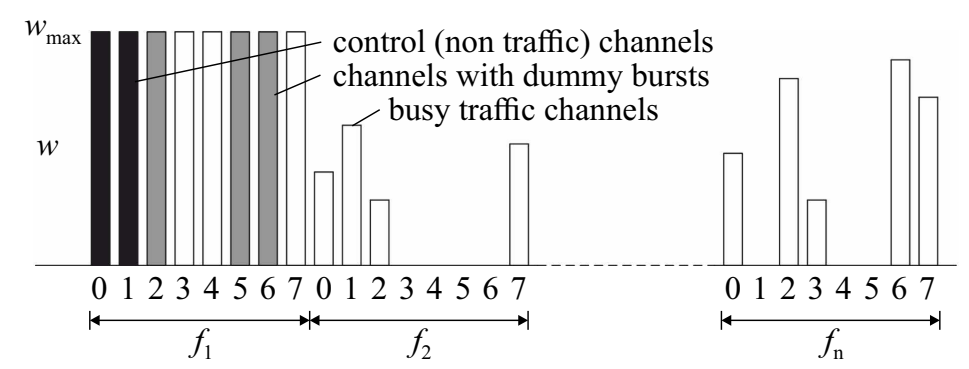

Fig. 1. Illustration of necessary emission power for GSM system time slots

environmental attenuation coefficient and the distance between user and BTS [3]. Although BTS power depends on more factors, in this paper it is supposed that it depends only on the distance between BTS and the user. It is supposed that the percent of intra-cell connections is negligible.

Mutual exchange of channels in realized connections after which the total transmitted power is less will be called reallocation. Channel reallocation will be carried out with the aim that the users who require greatest power take traffic channels in the first carrier. Channel reallocation is the process which is similar to intra-cell handover and it is not more complex from it. It is supposed that channel reallocation is so quick that users cant notice it.

The main considerations presented in this paper are related busy-hour, ie when it may be supposed that nearly all channels are busy and BTS emission power is greatest. The power will be designated by $w$, mean and maximum power of one channel will be designated by $w_{m}$ and $w_{\max }$, respectively. In this paper it is supposed that one channel emission power depends on user distance from BTS, $d$, according to the known expression $w=a \cdot d^{\gamma}$, where $a$ is coefficient of proportionality and $\gamma$ is transmission coefficient in some environment [13]. The typical value of $\gamma$ is between 2 and 5 [14]. It is also supposed that power which is transmitted to users changes continuously, and not as it is in praxis in steps of $2 \mathrm{~dB}$. The offered traffic is designated by $\mathrm{A}$ and call intensity (number of calls in the unit of time) by $\lambda$.

\section{Allocation of channels with power control}

As it is well known, the allocation of channels, ie time slots in GSM networks is as in Fig. 1. Each carrier has
8 time slots. First two time slots on the first carrier are signalling and remaining 6 are traffic. The total number of traffic channels is, therefore, $N=8 n-2$. BTS power of the first carrier and the power of all channels on the first carrier are always maximum nevertheless the channels are busy or not and this carrier is used as a beacon [15]. The power of the channels on the first carrier is symbolically presented in Fig. 1. Among them, channels 2, 5 and 6 are not busy. Let us consider the cell at the maximum traffic load, ie when all traffic channels are busy. In the cell with power control there are 6 traffic channels which have maximum power and remaining $8(n-1)$ traffic channels whose power is sufficient to realize the connection and dependent on distance between BTS and user. It is important to remark that first six traffic channels with maximum emission power are sometimes seized by users who need low emission power.

\section{Reallocation}

Let us now consider the same cell, but with the function of reallocation. Reallocation is the process of assigning the channels from the first carrier to users who need the greatest emission power. Reallocation may be realized at the connection beginning (when there is some idle channel between channels 2-7) or during the connection when some of the channels from the first carrier need lower power than some of the channels from the other carriers. In this second case the power difference between transmitted and necessary power in the channels of the first carrier is significantly lower than in the first case.

The simple example of channel reallocation in the cell with only two carriers is presented in Fig. 2. Figure 2(a) presents channels of both carriers before reallocation. The channels of the first carrier have maximum power, but the
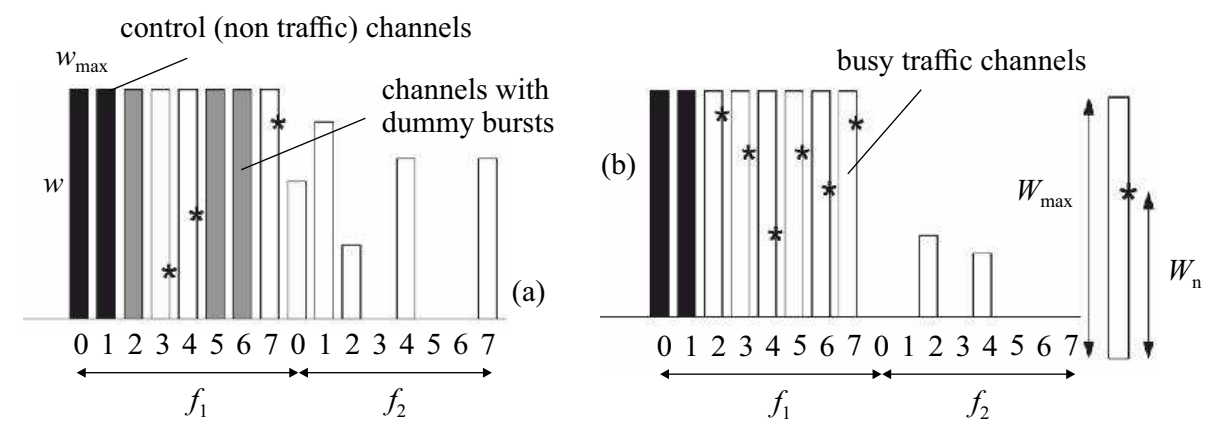

Fig. 2. Reallocation in the cell which has only two carriers 


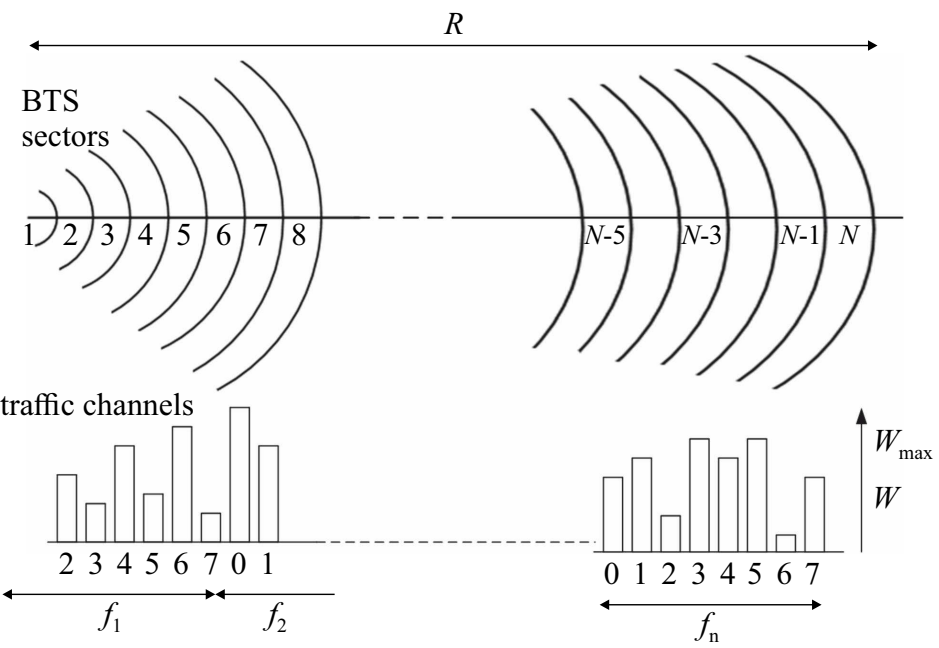

Fig. 3. Cell a: traffic process with power control without reallocation

asterisk on traffic channels designate what power would be sufficient for the realized connection $\left(w_{n}\right.$ is sufficient power). Some traffic channels on the first carrier are not busy, but they transmit "dummy" content in order to maintain maximum emission power on the first carrier.

Figure 2(b) presents the state on both carriers after reallocation. Traffic channels of the second carrier which need higher power are replaced by the channels of the first carrier. This means that the following replacements have been made:

channel 1 from $f_{2}$ is replaced by channel 2 in $f_{1}$, channel 4 from $f_{2}$ is replaced by channel 3 in $f_{1}$, channel 3 from $f 1$ is replaced by channel 4 in $f_{2}$, channel 4 from $f_{1}$ remains busy,

channel 7 from $f 2$ is replaced by channel 5 in $f_{1}$, channel 0 from $f 2$ is replaced by channel 6 in $f_{1}$, channel 7 from $f_{1}$ remains busy.

After the reallocation two consequences which influence at the total decrease of transmitted power may be seen:

- all traffic channels of the first carrier are busy,

- the channels of the first carrier are busy by the connections which need higher power and the channels of the second carrier by the connections which need lower power.

Reallocation is software defined process. It does require neither implementation of new hardware resources (channels) nor changes in existing hardware structures. Software for the reallocation realization would be very similar to the software for the simplest (intraBTS) handover. IntraBTS handover is explained in detail in [13], section 12.5.3.2. As also at this simple handover, reallocation is performed in the Base Station Controller (BSC), or even in Mobile Switching Centre (MSC). The conclusion is that modification of software to implement connections reallocation would not be expensive, because this software is not complicate and it is very similar to the existing software for handover.

\section{Estimation of power saving}

\subsection{Ideal case}

Transmitted power in each channel is random variable. That is why the powers are compared over their mean values. It is supposed that calls are generated in all network parts, ie that all distances between BTS and the user are possible and that all channels are busy. Two cells are considered: cell A with power control, Fig. 3, where each user can seize each channel and cell B with power control and reallocation, Fig. 4, where users with greatest power are placed in the first carrier and other channels are randomly engaged.

The mean necessary power of channel in the cell A, $w_{N m}$, is the mean power of all $N$ channels.

Reallocation is realized in the cell B. The most distant users from BTS who need greatest power are placed after reallocation in traffic channels of the first carrier, Figure 4. Assumption that users from 6 most distant sectors of the cell have seized 6 traffic channels on the first carrier makes this case ideal. The mean value of power in the channels which do not belong to the first carrier, $w_{(N-6) m}$, is calculated from the power of remaining $N-6$ channels. Since in this case 6 channels with the maximum power are isolated, it is clear that it must be $w_{N m}>w_{(N-6) m}$.

The total transmitted power in the cell $\mathrm{A}$ is

$$
w_{\mathrm{At}}=6 w_{1 \max }+(N-6) w_{N \mathrm{~m}} .
$$

The total mean transmitted power in the cell B is

$$
w_{\mathrm{Bt}}=6 w_{1 \max }+(N-6) w_{(N-6) \mathrm{m}} .
$$

As the first carrier power in both cells is maximum, ie equal, it is possible to compare the power of other carriers.

The power ratio of other channels in other carriers is

$$
G=\frac{w_{\mathrm{At}}}{w_{\mathrm{Bt}}}=\frac{(N-6) w_{N \mathrm{~m}}}{(N-6) w_{(N-6) \mathrm{m}}} .
$$

Factor $G$ may be considered to be an indicator of energy savings. 


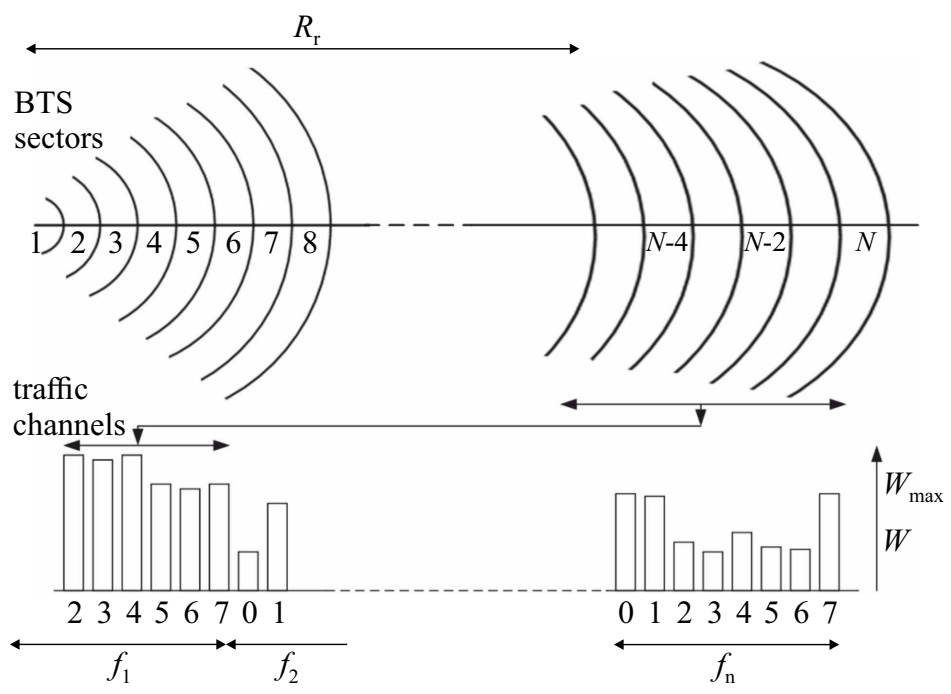

Fig. 4. Cell b: traffic process with power control and reallocation(ideal case)

\subsection{Real case}

Unfortunately, ideal case occurs only from time to time during traffic process and it is not significant to estimate energy of the cell with reallocation. The most common case of real traffic is the one when traffic grows and there are often more than 6 connections of users from the most distant sectors who increase mean power in the cell B with reallocation. In this case (2) becomes

$$
w_{\mathrm{Bt}}=6 w_{1 \max }+(N-6) w_{(N-6) \mathrm{m} 2},
$$

where $w_{(N-6) m 2}$ represents mean power of the remaining $(N-6)$ channels. Among these channels some of them require high power and the consequence is that it is $w_{(N-6) m 2}>w_{(N-6) m}$ and the power saving is lower than in the ideal case.

\section{An example}

Ideal and real case will be investigated by the following numerical example: $N=14, n=2, \gamma=2$, and the uniform surface user density distribution in the cell. According to (8) from [2], it may be proved that the mean power per a user is half a maximum power, ie $w_{m}=0.5 w_{1 \max }$, $w_{1 \text { max }}=a R^{2}$ for uniform user surface distribution.

In ideal case the mean channel power for carriers other than the first one in the cell A without reallocation equals

$$
w_{\mathrm{Nm}}=0.5 w_{1 \max }=0.5 a R^{2} .
$$

Ideal case in the cell B implies that 6 most distant users have seized 6 traffic channels in the first carrier, Fig. 4. Other traffic channels on other carriers except the first one are seized by users from closer segments of the cell. The closer segments form the cell/circle with the radius
$(8 / 14) R$. The mean power of one channel in such a cell B is

$$
\begin{aligned}
& w_{(N-6) t m}=0.5 w_{1} \max r=0.5 a\left(\frac{N-6}{N} R\right)^{2} \\
&=0.5 a\left(\frac{8}{14} R\right)^{2}=0.3265 w_{N m}
\end{aligned}
$$

where $w_{1 \max r}$ is maximum power of the channel in a small circle and it is equal a $(8 / 14)^{2} R^{2}$.

The power of the second carrier in the cell $\mathrm{A}$ is

$$
w_{A}=(N-6) w_{N m}=4 w_{1 \max },
$$

and in the cell B

$$
\begin{aligned}
w_{B}=(N-6) & w_{(N-6) m} \\
= & 0.3265(N-6) w_{N \mathrm{~m}}=1.306 w_{1 \max }
\end{aligned}
$$

thus causing that the power ratio of second carrier in the cells A and B is about 3.06 .

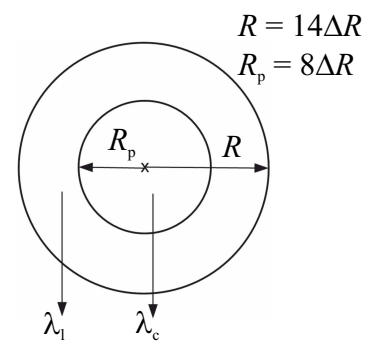

Fig. 5. Traffic process in the cell which is divided in closer and further segments

The real case in this cell includes traffic process randomness and possibility of traffic increase. Traffic randomness is reflected in the different proportions of calls from near and distant parts of the cell: their balance is reflected only in the mean values. In order to see what 
it is, let us imagine that a cell is divided in 8 closer and 6 further segments, Fig. 5 .

It may be proved that the number of users in the circle (inner area) is $32.64 \%$ and in the ring (outer area) is $67.36 \%$ of total user number in the cell. The same is the offered traffic ratio from two cell parts, ie $\lambda c=0.3264 \lambda$ and $\lambda_{l}=0.6736 \lambda$. Let us suppose that unit of time is equal to the mean conversation/connection time. In that case offered traffic is equal to the call (traffic) intensity, ie $A=\lambda$. On the base of Poissonian distribution implementation it may be concluded that there is real probability for more than 6 call requests to come from the outer ring in short time for the offered traffic of $A=10 \mathrm{Erl}$ : $P(7)=0.1482 ; P(8)=0.1247 ; P(9)=0.0933$, etc. This means that more distant users will seize also some channels of the second carrier besides the 6 channels of the first carrier, thus decreasing the reallocation contribution to the power saving. Let us suppose that two users from the distant sectors appear in the channels of the second carrier. The effect is the same as that inner area Fig. 5, is expanded from 8 to 10 segments due to increased offered traffic. The calls from these segments seize channels both from the first and from the second carrier. The power necessary to the new circle, ie to the channels in the second carrier is now

$$
w_{B}=4 a\left(\frac{10}{14} R\right)^{2}=1.96 w_{1 \max }
$$

and this is two times less than the carriers without reallocation $\left(4 w_{1 \max }\right)$.

This numerical example is important, because the adopted traffic value $(10 \mathrm{Erl})$ is real for 14 available channels because the corresponding traffic loss is about $5 \%$. The calculation results are verified in $[2-4]$ by the simulation programs for communication process. These programs are adjusted to estimate emission power in mobile network cell. As a contribution of this paper it is additionally developed the simulation program for connections reallocation. The calculation results from this paper may be verified applying our originally developed simulation program which includes reallocation and the simulation program without reallocation. The simulation in twenty trials gives the mean value $w_{B \text { simul }}=2.025$ for the emphasized numerical example, ie that the mean value is in the range 2.022-2.027 with the probability 0.95 . Difference between the results of calculation and simulation is explained in the following way: reallocation, ie power variation during the connection life-time, is hardly calculated, but may be easier determined in the simulation. That is why power is calculated according to its value at the connection beginning, not considering later variations.

The consequence of traffic increase is that more connections appear from all mobile network cell segments. This is the reason why more requests appear also from distant network parts, causing the increased competition for 6 channels from the first carrier. There are more and more connections with the increased power, which have to be realized over the channels from the second, third or other carriers. In this way we may explain how traffic increase leads to lower power saving (besides traffic loss increase). The behaviour is illustrated by the previous numerical example. Figure 6 presents power ratio in the model without reallocation (wA) to the model with reallocation (wB) as a function of offered traffic $(\mathrm{A})$. The results are obtained by simulation.

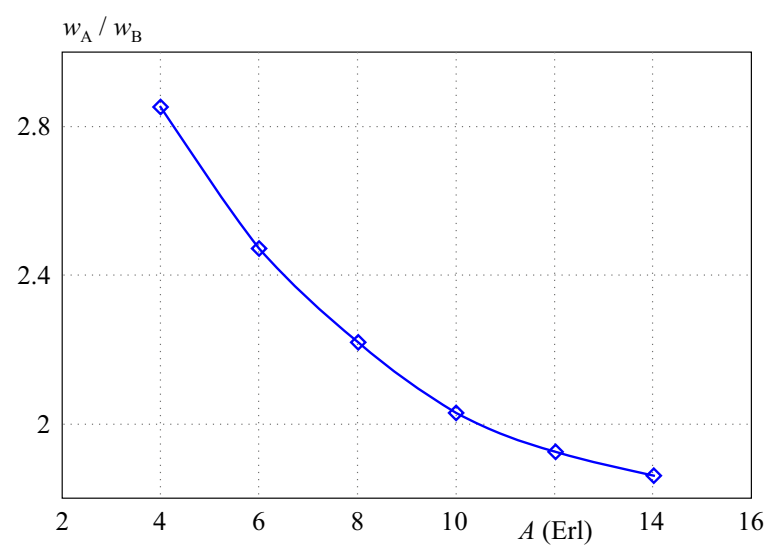

Fig. 6. Power ratio between the models without reallocation $\left(w_{a}\right)$ and with reallocation $\left(w_{b}\right)$ as a function of offered traffic $(\mathrm{A})$

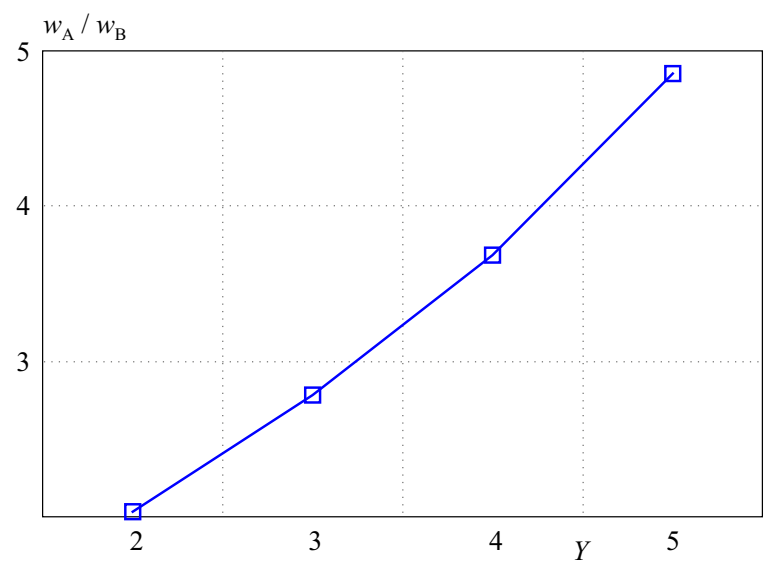

Fig. 7. The ratio of the necessary channel power in the cell A (without reallocation) to the cell B (with reallocation) as a function of environmental propagation coefficient $(\gamma)$

Table 1. The ratio of the necessary channel power (excluding the first carrier) in the cell A (without reallocation) to the cell B (with reallocation) as a function of offered traffic (A Erl)

\begin{tabular}{lccc}
\hline$N$ & 14 & 22 & 30 \\
\hline$A(\operatorname{Erl})$ & 10 & 17 & 25 \\
$w_{A} / w_{B}$ & 2.025 & 1.51 & 1.315 \\
\hline
\end{tabular}

\section{Power decrease as a function of the number of carriers, environmental propagation coefficient and user distribution in the cell}

\subsection{Dependence on the number of carriers}

It is logical to expect that the increase of the number channels/carriers causes power saving decrease, because 
Table 2. The ratio of the necessary channel power in the cell A (without reallocation) to the cell $\mathrm{B}$ (with reallocation) for three different user distribution types

\begin{tabular}{lccc}
\hline $\begin{array}{l}\text { User density } \\
\text { distribution }\end{array}$ & Decreasing & Uniform & Increasing \\
\hline$w_{A} / w_{B}$ & 2.52 & 2.025 & 1.76 \\
\hline
\end{tabular}

the number of channels for the connections with greatest power remains always the same. The ratio of the necessary channel powers (excluding the first carrier) in the cell A (without reallocation) and the cell B (with reallocation) is presented in Tab. 1. The other conditions are: $\gamma=2$, user surface density distribution is uniform and traffic loss is about $5 \%$.

\subsection{Dependence on the environmental propagation coef- ficient}

The increase of environmental propagation coefficient $(\gamma)$ value causes the increase of necessary power difference between the cell A (without reallocation) and the cell B (with reallocation), Fig. 7. User density distribution in both cells is uniform.

\subsection{Dependence on the user density distribution in the cell}

Three cells with specific distributions of user surface density may be considered, as in [2]: the cell with decreasing user density distribution from the BS (density 6) towards the cell rim (density 1 ), the cell with uniform user density distribution and the cell with increasing user density distribution from the BS (density 1 ) towards the cell rim (density 6). The cell with two carriers, offered traffic $A=10 \mathrm{Erl}$ and environmental propagation coefficient $\gamma=2$ will be considered in all three cases. Two different cells are analyzed for each user density distribution: the cell A without channel reallocation and the cell B with channel reallocation. As in the previous cases, the ratio $w_{A} / w_{B}$ is determined. The obtained results are presented in Tab. 2.

In ideal case this ratio is about 3 . Unfortunately, calls from distant users appear very often (especially when user density distribution is increasing which is in praxis, fortunately, rare). Calculating a part of total calls number from the remote segments of the cell when increasing user density distribution is analyzed, it is obtained, at the assumed parameter values, that a part of new calls from the remote 6 segments is $\lambda_{l}=0.835 \lambda$ and from close ones $\lambda_{c}=0.165 \lambda$. The probability that more than 6 calls appear in short time interval from the most distant segments of the cell is even $P(>6)=0.7027$. It means that all calls which require greatest power cannot be placed in the channels of the first carrier, but always seize several channels on the second carrier (in this example: 2 or 3 ).

It is important to emphasize that Tab. 2 presents relative power ratio. Emission power saving in absolute rate may be higher at increasing or uniform user density distribution because the majority users are more distant from BS than in the cell with decreasing user density distribution.

\section{Simulation}

The simulation which determines emission power ratio in the cell without reallocation to the power of cell with reallocation uses modified program from [2]. Roulette or Monte Carlo simulation is the fundamental principle implemented in the simulation [16]. The situation without reallocation is simulated taking the simulation program from [2]. The supplement to this program is the part which performs channel reallocation according to the required emission power. The new part of simulation program is the contribution of the paper. In this simulation channels are arranged in order of their distances from the base station, starting from the largest towards the smallest distance. At the time of each new call generation reallocation is performed such that ordinal number of the new user in the conversation phase is determined in relation to the existing connections. Besides a random number which defines that it is necessary to start a new call, another, additional, random number that defines the distance from the base station is also generated. All users with a lower distance increase for 1 their ordinal number in relation to the situation before the new call generation, and the new call with its distance is placed at the vacant place in series. The total number of different distances is increased for 1 in this way. The reallocation is also performed in the moment when the connection is terminated. The value of the generated random number determines the channel number on which the connection is ended. All distances in a series, which are after the channel determined by the generated random number, get now for 1 lower ordinal number in relation to the situation before the connection termination. In this manner the old distance for the channel on which the connection is terminated gets lost. The channel, which was before the connection end the last in the series, gets the value of a distance equal to 0 . In this way the total number of different distances is decreased for 1 .

\section{Conclusion}

Channel reallocation may be always implemented in GSM mobile network cells with power control to save emission power. It is important to respect two principles. The first one is to use channels from the first carrier when the offered traffic is low. The second and more important one is that users who require greatest emission power use channels from the first carrier after reallocation. Channel reallocation is not more complicate than the simplest handover procedure. There is no connection quality degradation caused by the increased signal delay and loss, as in [12]. Power saving is relatively higher for low number of carriers, for decreasing user surface 
density distribution in the cell and for higher values of environmental propagation coefficient. Numerical examples prove that power saving may be from several tens to several hundred percent.

\section{REFERENCES}

[1] R. Švraka, D. Mitić, A. Lebl and Ž. Markov, "Calculating Limits of Base Station Emission Power GSM", Automatika, vol. 57 , no. 3, pp. 774-781, Sep 2016, DOI: 10.7305/automatika. 2017.02.994.

[2] M. Mileusnić, M. Popović, A. Lebl, D. Mitić and Ž. Markov, "Influence of Users' Density on the Mean Base Station Output Power", Elektronika ir Elektrotechnika, vol. 20, no. 9, pp. 74-79, Nov 2014, DOI:https://doi.org/10.5755/j01.eee.20.9.5418.

[3] P. Jovanović, M. Mileusnić, A. Lebl, D. Mitić and Ž. Markov, "Calculation of the Mean Output Power of Base Transceiver Station GSM", Automatika, vol. 55, no. 2, pp. 182-187, June 2014, DOI: https://doi.org/10.7305/automatika.2014.06.373.

[4] D. Mitić, A. Lebl and Ž. Markov, "Influence of Traffic Model on the Calculation of BTS Output Power GSM Network, AEÜ", International Journal of Electronics and Communications, vol. 69, no. 5, pp. 836-840, May 2015, DOI: https://doi.org/10.1016/j.aeue.2015.02.003.

[5] A. Lebl, D. Mitić, B. Trenkić and Ž. Markov, "Determination of Base Station Emission Power Change a Mobile Network Cell with Movable Users", Radioengineering, vol. 27, no. 4, pp. 1174-1182, Dec 2018, DOI: 10.13164/re.2018.1174.

[6] V. E. Beneš, "Mathematical Theory of Connecting Networks and Telephone Traffic", Academic Press, New York, 1965.

[7] O. Lemeshko and O. Yeremenko, "Enhanced Method of Fast Re-Routing with Load Balancing Software-Defined Networks", Journal of Electrical Engineering, vol. 68, no. 6, pp. 444-454, Dec 2017, DOI: 10.1515/jee-2017-0079.

[8] A. Lebl, D. Mitić, Ž. Markov, M. Stanić and M. Mileusnić, "A Decrease of Unsuccessful Pairing Probability VAMOS Mobile Cell Using Existing Connection Rearrangement", Technical Gazette, vol. 27, no. 1, pp. 106-113, Feb 2020, DOI: 10.17559/TV-20180703100537.

[9] W. B. Iversen and A. J. Glenstrup, "Resource Allocation Cellular Wireless Systems", J. Woniak, J. Konorski: "Personal Wireless Communication", IFIP TC6/WG6. 8 Working Conference on Personal Wireless Communications (PWC'2000), pp. 123-132, 14-15th Sep2000., Gdaňsk, Poland, Kluwer Academic Publishers Group.

[10] T. Jensen, "Performance Analysis of Multilayer Cellular Networks with Classes of Mobile Stations", J. Labetoulle, J. W. Roberts, The Fundamental Role of Teletraffic the Evolution of Telecommunications Networks, vol. 1a: Teletraffic Science and Engineering, Elsevier, pp. 481-492, 1994.

[11] V. B. Iversen, V. Benetis and P. D. Hansen, "Performance of Hierarchical Cellular Networks with Overlapping Cells", G. Kotsis, O. Spaniol, Wireless Systems and Mobility Next Generation Internet, First International Workshop of the EURO-NGI Network of Excellence, Dagstuhl Castle, Germany, pp. 7-19, 7-9 June 2004, Revised Selected Papers, Springer.

[12] S. Suherman, N. Mubarakah and M. Al-Akaidi, "Minimizing Energy Consumption on Mobile Phone by Rearranging Transport Protocol Load", International Journal of Engineering \& Technology, vol. 7, no. 3, pp. 713-717, July 2018, DOI: 10.14419/ijet.v7i3.2.15350.

[13] G. Heine et al, "GSM Networks: Protocols, Terminology and Implementation", Artech House, 1999.

[14] J. Ebersprächer, H. J. Vögel and C. Bettstetter, "GSM, Switching, Services and Protocols", John Wiley \& Sons, Sep 2001,.
[15] E. Seurre, P. Savelli and P. J. Pietri, "GPRS for Mobile Internet", Artech House Boston London, 2003.

16] L. Kosten, "Simulation Teletraffic theory", 6th ITC, pp. 411/1-411/8, Munich 1970.

Received 2 February 2020

Vladimir Kosjer was born in Indija, Serbia, in 1989. He received his BSc and MSc from the Faculty of Technical Sciences in Novi Sad, Republic of Serbia, in 2012. and 2016., respectively, and started $\mathrm{PhD}$ studies in 2019. at the same Faculty. During 2014/15. he was employed in EBV as Field application engineer, and from 2015-2019. in RT-RK National research institute as software/firmware developer on different projects, eg 3D surround sound, Android based smart STB devices and in automotive segment, driving assistance. During the period of 2 school years, he was employed as teaching Assistant at the Faculty of Technical Sciences. He is currently employed in IRITEL in Radio communication department.

Dragan Mitić was born in Belgrade, Serbia, in 1953. He received his BSc and MSc from the Faculty of Electrical Engineering in Belgrade, Republic of Serbia, in 1977 and 1984, respectively, and his $\mathrm{PhD}$ from the Faculty of Technical Science in Novi Sad, in 2002. He is a senior research associate in IRITEL, Institute for Electronics and Telecommunications, Belgrade, Serbia. From 1977. Until 1989 he was employed at the Land Forces Military Technical Institute in Belgrade, and since 1989. in IRITEL. Dr Miti is author or co-author of more than 200 international and national scientific and professional papers. He works on several research projects for equipment of specific applications.

Aleksandar Lebl was born in Zemun, Serbia, in 1957. He received his BSc and MSc from the Faculty of Electrical Engineering in Belgrade, Republic of Serbia, in 1981 and 1986, respectively, and his $\mathrm{PhD}$ from the Faculty of Technical Science in Novi Sad, in 2009 he was employed from 1981 in the Switching Department of Institute for Electronics and Telecommunications IRITEL in Belgrade. During years he worked on the project of Digital Switching System for Serbian Telecommunication Industry. From 2015 he works in Radio communications department, also in IRITEL.

Vladimir Matić was born in Belgrade, Serbia, in 1963. He received his BSc and MSc from Faculty of Electrical Engineering in Belgrade, Republic of Serbia, in 1989 and 1994, respectively and his $\mathrm{PhD}$ from the Faculty of Technical Science in Novi Sad, in 2016. From 1996 he is employed in Radio communications department of Institute for Electronics and Telecommunications IRITEL in Belgrade, where now holds the position of project leader. He published more than 50 scientific papers in international and national journals and conferences and participated in realization of several scientific and research projects of the Ministry of Science and Technology of Republic of Serbia and Institute IRITEL.

Žarko Markov was born in Žitište, Serbia, in 1946. He received his BSc, MSc and $\mathrm{PhD}$ from the Faculty of Electrical Engineering in Belgrade, Republic of Serbia, in 1969, 1975 and 1976, respectively. He is a scientific counsellor in IRITEL, Institute for Electronics and Telecommunications, Belgrade, Serbia. Area of work: Switching technics, Teletraffic theory, Network signalling. Author or co-author of more than hundred papers and six books. At the University of Belgrade, School of Electrical Engineering, He was a professor at the course of Switching technics and Network signalling. 